\title{
Special Issue: The Sustainable Groundwater Management Act
}

T his special issue of California Agriculture focuses on the implications of the Sustainable Groundwater Management Act, or SGMA, the package of bills signed into law by California Gov. Jerry Brown in September 2014.

The legislation puts California on a path to the sustainable management of a resource that accounts for between one-third (in wet years) and two-thirds (in dry years) of the state's water use. In dry years, statewide overdraft is in the range of 3 to 7 million acre-feet. Some of that is recharged in wet years, but in many basins, including some of the state's top-producing agricultural regions, the long-term trend has been falling water tables (see groundwater.ucdavis.edu for links to UC video introductions to groundwater in California and SGMA).

Sustainable management under the act is defined as the avoidance of "significant and unreasonable" levels of six impacts: (1) lowering of groundwater levels, (2) reduction in groundwater storage, (3) seawater intrusion, (4) water quality degradation, (5) land subsidence and (6) impacts on beneficial uses of interconnected surface waters.

Regulation of groundwater in California was long in coming. The state has often been a national leader in environmental policy, for instance on air quality and greenhouse gas emissions. But it was the last state to adopt statewide groundwater regulations (Cannon Leahy 2016). In most basins, groundwater use was unrestricted beyond the basic requirement in the state constitution that all water use be reasonable and beneficial.

Of the state's 515 groundwater basins, SGMA applies to the 127 judged to be high- or medium-priority based on a combination of factors related to current and future groundwater demand and the consequences of overdraft. These 127 basins account for $96 \%$ of the state's groundwater extraction (CASGEM 2018). Twenty-seven of these basins have previously been adjudicated - meaning that they are under a court order to limit overdraft.

The act mandated the creation, by June 30, 2017, of a new set of local agencies, groundwater sustainability agencies (GSAs), responsible for complying with SGMA's directives. To date, 266 groundwater sustainability agencies have been formed, accounting for 378 areas in 141 basins statewide (in many cases there is more than one GSA in a basin; also, GSAs may be formed in low-priority basins even though the law does not require it).

The next step is the preparation of groundwater sustainability plans. These plans must put the basin on a path that will lead to sustainable management within 20 years, with interim milestones subject to state review every 5 years. Critically overdrafted basins (map) must submit plans by January 31, 2020; other basins must submit by January 31, 2022.

Two state agencies have primary responsibility for supporting the implementation of SGMA. The Department of Water Resources acts as facilitator and evaluator, while the State Water Resources Control Board has an enforcement role, ensuring compliance with the law (see article page 18).

California Agriculture thanks the guest editors of this special issue: Faith Kearns and Doug Parker (California Institute for Water Resources, UC Agriculture and Natural Resources), Meredith Niles (University of Vermont) and Kurt Schwabe (UC Riverside). The journal also thanks the Stockholm Environment Institute for supporting a portion of the printing costs of the issue. CA

The hydrologic cycle. Source: California Department of Water Resources, Water Budget Best Management Practice, December 2016.
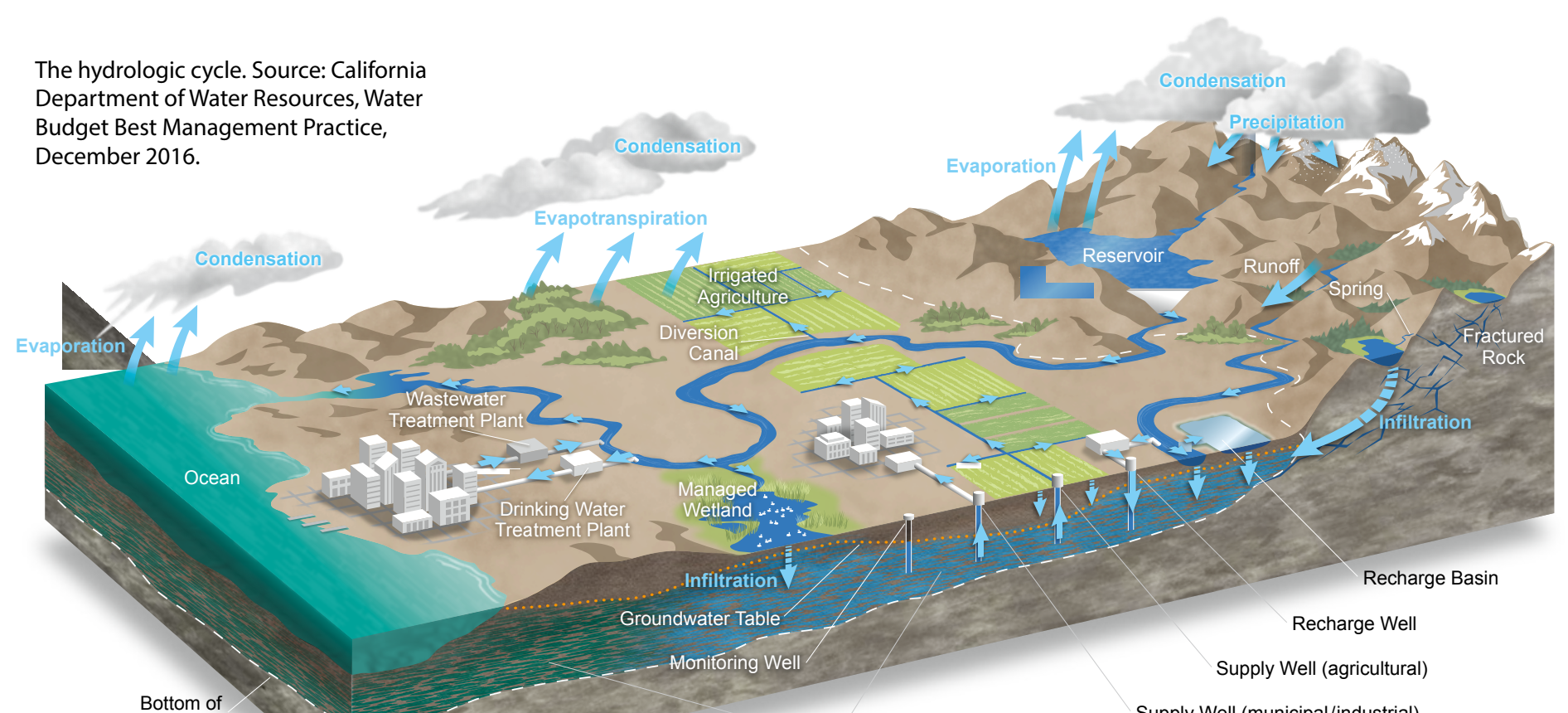

Supply Well (municipal/industrial) 


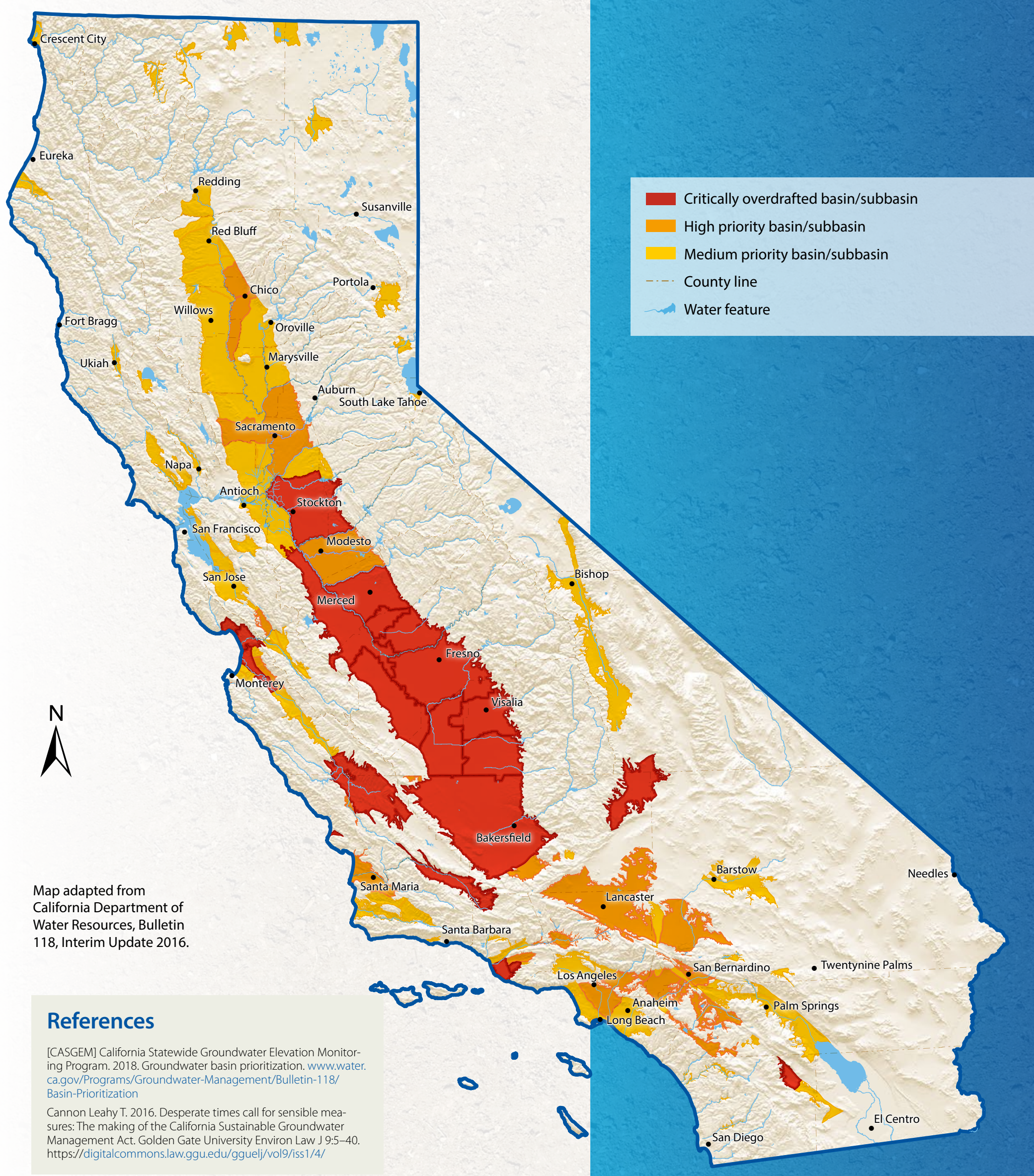

